\title{
DE LA METODOLOGIA Y LAS ESPECIES DIMISIONARIAS
}

\author{
José Luis Crespán Echegoyen \\ (Universitat Autònoma de Barcelona)
}

Si existe algún fantasma que recorre los campos sociológicos, no cabe la menor duda que es el fantasma de la metodología. Martillo de herejes y refugio de pecadores, matrona inflexible y cortesana complaciente a la vez, la metodología se ha visto entronizada en el lugar principal de cada casa. La crisis, o mejor, las crisis que sacuden al cuerpo sociológico desde hace tiempo, han provocado la emergencia de un territorio sacralizado del que nadie discute su existencia y necesidad, pero acerca del cual no se consigue un mínimo acuerdo en lo referido a su propia definición y consistencia: es el territorio de la metodología.

Asistimos, pues, más o menos perplejos, más o menos combativos, al momento estelar de un llamado debate metodológico en el que las más de las veces se vuelcan argumentos impertinentes traídos de otros ámbitos de debate. Ello provoca desasosiegos varios entre el personal y se traduce frecuentemente en descalificaciones y denostaciones genéricas respecto de la metodología y de los metodólogos.

Sea como fuere, la denostación corre pareja a la fascinación. Porque la metodología, como dama frívola y esquiva, como personaje de mil caras, seduce y abandona. Habría otta lectura posible de la metodología como autoseducción, como espejo, y en la que cada uno propone exactamente como modelo aquello que uno hace o es. De esta manera nos encontraríamos, además de inmersos en un juego de narcisos silenciosos, ante la imposibilidad manifiesta de comunicar a los demás nuestra vetdad o nuestra belleza $\rightarrow$ pata el caso es to mismo-, pues serían absolutamente incapaces de 
comprenderla y compartitla absolutamente seducidos por la suya propia. Pero dejaremos este apunte de lectura para otra ocasión. ${ }^{\text {t }}$

Cabe legítimamente preguntarse cómo puede haberse llegado a una tal situación de ambivalencia y, sobre todo, preguntarse cómo podemos encontrar la tangente de escape a este circulo infernal, que si bien no es seguro que nos vaya a conducir al paraíso sin escalas intermedias, sí por lo menos nos pueda proporcionat una nueva perspectiva de la cuestión $\mathrm{y}$, por lo tanto, una posibilidad de redención.

Con respecto a la primera pregunta hay que resaltar que la metodología (permítasenos este singular impropio, pues sería más adecuado hablar de metodologías o de prácticas metodológicas) ha ocupado este lugar central y privilegiado en la medida que ejerce una triple función que en otras disciplinas más consolidadas que la Sociología ha ejercido y ejerce la teoría. En efecto, la metodología ejerce, en el contexto de las ciencias sociales, una primeta función de legitimación-exclusión. En una situación generalizada de incertidumbre cognoscitiva y normativa, el sistema de exclusiones, de rechazos, queda exclusivamente asignado a la dicha metodología.

En segundo lugar, la metodología aparece como la garantía de «estar en verdad». Es ésta una función complementaria de la anteriot hasta el punto que, aun siendo diferentes, la una no tendría sentido pleno sin la otra. No sólo se trata de expulsar a los falsos, sino además de garantizar nuestra verdad.

$Y$, finalmente, la metodología tal y como hoy está conceptualizada, ejerce lo que podríamos llamar la función de control de la producción del discurso sociológico. Es ésta una función de segundo grado que no opera tanto a nivel de sistema normativo estricto como a nivel de la negación de la posibilidad de producciones intelectuales anómalas o transdisciplinares. De alguna manera esta función no hace sino enmascarar una patente fobia interdisciplinar, haciendo aparecer una aparente y exclusivamente verbal filia transdisciplinar.

La posibilidad del ejercicio de esta triple función se ha producido a partir de una situación crítica del sistema científico que afecta no tan sólo a las ciencias sociales, conceptualizadas por algunos como de desarrollo preparadigmático, sino a la práctica globalidad de las ciencias. Esta crisis científica viene caracterizada por una triple dimensión: 1) un cierto agotamiento del paradigma científico dominante, 2) la quiebra de la hegemonía

1. Cuando estábamos dando los últimos toques a este escrito, ha Ilegado a nutes tras manos un magnífico ejemplar de esta lectura narcisista: «Consideracions sobre la crisi actual de la Sociologia», de J. Estruch y S. Cardús, en Butlletí Informatiu de la Fundacio Jaume Bofill, Barcelona, 1984. Tal y como decinos en el texto, dejamos su comentario para posterior ocasión. 
del modelo disciplinar y 3) la revisión de la relación sujeto-objeto en las prácticas del conocer.

Conviene que nos entretengamos un mínimo en cada una de ellas para poder explicar la ascensión a la centralidad de la metodología y, particular. mente, qué metodología es la que se constituye como central.

\section{Agotamiento del paradigma cientifico dominante}

Pasando por encima de la polisemia del término paradigma, del que, como ha señalado Masterman, ${ }^{2}$ el propio Kuhn hace más de una veintena de usos diferentes, y reteniendo exclusivamente el concepto de modelo o ejemplo pautado, detectamos en el desarrollo científico actual la aparición de indicios más que suficientes para entender que nos hallamos a las puertas de una fase de lo que Kuhn ${ }^{3}$ denomina «ciencia extraordinaria».

Resulta probada la emergencia de preguntas impertinentes a las que los científicos «ordinarios» no pueden dar respuesta $o$, incluso, no pueden formularlas en términos operativos por estar ubicadas en un contexto no significativo para el modelo con el que operan. Lo cual no es reducible a decidir la ilegitimidad de las preguntas en base a ctiterios de contenido de la ciencia "ordinaria» o en base a criterios de adecuación del sujeto o sujetos sociales que las formulan, como todavía se postula desde numetosos ámbitos corporativos. La posibilidad de formulación operativa y, por ende, de respuesta a tales preguntas obliga a operar en términos post-paradigmáticos con lo que Lakatos ${ }^{4}$ ha denominado «lógica de descubtimiento", que no siempre pretende la formulación de un paradigma de tipo superior como pretende decir una cierta historia natural de la ciencia.

Todo ello viene a demostrar la imposibilidad manifiesta de una ciencia «extraordinaria» como desartollo paradigmático de la ciencia "ordinaria» y obliga a abandonar la vieja ilusión de una normativa estricta aplicable a la resolución de no importa qué problema del ámbito disciplinar cortes. pondiente.

Esto plantea la incómoda disyuntiva de, o bien aceptar la incapacidad del paradigma con su metodología incluida, o bien aceptat que el mencio-

2. Masterman, Margaret: 1975, «La naturaleza de los paradigmas», en Lakatos y Musgrave (eds.), La critica y el desarrollo del conocimiento, Barcelona, Grijalbo, pp. $159-201$.

3. Kuhn, Thomas S.: 1975 (e.o. 1902), La estructura de las revoluciones cientifi. cas, Madrid, Fondo de Cultura Economica.

4. Lakatos, Imre: 1974, «Metodologías rivales de la ciencia: las construcciones sacionales como guía de la historia», en Teorema, vol. IV/2, pp. 199-214. 
nado paradigma es sólo una reconstrucción parcial de los objetivos y procedimientos utilizados por los hombres en sus prácticas de conocer.

En cualquier caso, el dilema sólo tiene sentido en y desde un posicionamiento paradigmático y la imposibilidad de una resolución satisfactoria apunta claramente en la dirección del agotamiento antes mencionado del modelo dominante.

Para nosotros resulta mucho más sugerente, y también eficiente, seguir la propuesta de Feyerabend ${ }^{5}$ de «sustituir los castillos formales en el aire, bellos pero inútiles, por un estudio detallado de las fuentes primarias en la historia de la ciencia», que, de alguna manera, entronca con la pretensión foucaultiana de una arqueologia del saber.

\section{Quiebra de la begemonia del modelo disciplinar}

Tradiciorialmente las diferentes disciplinas científicas se han construido y consolidado como resultado de una apropiación en exclusiva de ciertos objetos de conocimiento por parte de científicos que, necesariamente, se han visto obligados a formular o reformular un método propio e interno a la disciplina.

Además de una posible lectura de la disciplinarización del conocimiento como manifestación de las prácticas de poder social de los científicos, tal y como dice Foucault, ${ }^{6}$ cabría destacar con Cerroni ${ }^{7}$ lo que tal proceso ha supuesto de «desarticulación del objeto» y de «pulverización del método». Desarticulación del objeto en la medida que su instauración como elemento definitorio de la disciplina supone, voluntaria o involuntariamente, su desarraigo del contexto, que es el que, precisamente, ha revelado al objeto como significativo y, en consecuencia, supone una pérdida notable de dimensiones sustantivas. Por otra parte, la exigencia de privatización de un método, las más de las veces sólo justificable como criterio de demarcación-exclusión legitimadora, supone una alteración profunda de una de las características del método científico, a saber, su pretensión de universalidad.

Pero, al margen de esta lectura del proceso de disciplinarización de la ciencia, hay que consignar otro fenómeno que es el que precisamente pone en cuestión la hegemonía de la disciplina como unidad estructurante de la

5. Feyerabend, Paul K.: «Filosofía de la ciencia: una materia con un gran pasado», en Teorema, vol. IV/1, pp. 11-27.

6. Foucault, Michel: 1970 (e.o. 1969), La arqueología del saber, México, Siglo XXI.

7. Cerroni, Umberto: 1971 (e.o. 1968), Metodología y ciencia social, Barcelona, Martínez Roca. 
ciencia. Tal fenómeno no es otro que lo que podemos denominar la «revuelta de los objetos». En efecto, los objetos de conocimiento se constituyen cada vez más como objetos contextuales, teórico-abstractos, con la consecuente pérdida de su inmediato referente material.

Esta constitución de los objetos de conocimiento, que ya había sido significada por Weber ${ }^{8}$ y otros autores en el terreno de las ciencias sociales, empieza a ser confirmada por científicos de las ciencias naturales como Prigogine ${ }^{9} \mathrm{y}$ otros.

De algún modo, esta transformación desplaza el criterio de demarca. ción de los objetos a los procedimientos de conocer tales objetos. La práctica científica disciplinar se ve obligada a utilizar como elemento central de estructuración la llamada metodología, tal y como hemos dicho anteriormente. Pero resulta obvio que éste es un criterio de exclusión mucho más laxo y, sobre todo, obliga a los científicos disciplinares a una práctica esquizofrénica en la que simultáneamente se ha de legitimar la validez universal del método científico y la existencia y necesidad de un método particulat de la correspondiente disciplina.

Es precisamente por este sesgo por donde empiezan a aflorar diferentes conceptualizaciones de la metodología según sea pata uso interno de los miembros de una disciplina o para uso externo frente a otros colectivos.

\section{La revisión de la relación sujeto-objeto en las prácticas del conocer}

El desarrollo del conocimiento científico, al menos del que constituye nuestra referencia temporal, se basa en el axioma innegociable de la existencia de una realidad externa al sujeto, verificable por no importa quien, siempre que utilice los mecanismos adecuados de observación.

El modelo experimental supone la posibilidad de reproducción de situaciones exactamente iguales el númeto deseado de veces por parte del investigador. Ello implica la definición de una externalidad absoluta del objeto de conocimiento con respecto al sujeto que lo realiza. La situacion paradigmática es la de absoluta descontaminación del científico respecto de los objetos que estudia. La subjetividad, es decir, cualquier vinculación emo-

8. Weber dice textualmente: "No son las relaciones reales entre "cosas" lo que constituye el principio de delimitación de los diferentes campos científicos, sino las relaciones conceptuales entre problemas.» Weber, Max: 1965, Essais sur la théorie de la science, Patís, Plon.

9. Prigogine, Ilya y Stengers, Isabelle: 1983 (e.o. 1979), La nueva alianza, Madrid, Alianza Editorial. También puede verse Prigogine, Ilya: 1983, «२Tan sólo una ilusión?», Cuadcrnos Infimos, 111, Batcelona, Tusquets. 
tiva entre sujeto y objeto, debe ser eliminada, puesto que constituye, según el paradigma, un obstáculo epistemológico de primet orden.

Pues bien, hoy nos encontramos que, en la práctica científica, la experimentación, tal y como viene definida paradigmáticamente, está perdiendo su carácter de centralidad procedimental. Y ello por diversas razones. En primer lugar por el propio carácter de los objetos, que, además de su condición relacional teórico-abstracta, se sitúan en microescalas o macroescalas de imposible contrastación empírica por la vía de la experimentación convencional. Pero es que además estas condiciones abren la puetta a un hecho, tradicionalmente denostado en la práctica científica, como es la intetpretación.

Nos encontramos así ante un hecho relativamente novedoso como es el de que todo proceso de producción de conocimientos es, sobre todo, un proceso de interpretación o, lo que es lo mismo, un proceso de producción simbólica sólo significable y comprensible a partir de la condición de histo. ticidad del sujeto que lo desarrolla.

La coattada de una realidad exterior, reproducible y experimentable, independiente del sujeto ha comenzado a fracasar de manera generalizada y no sólo en los campos científicos sociales donde siempre había planteado problemas importantes, sino también en los terrenos científicos naturales, tal y como han puesto de manifiesto Prigogine y Wagensberg ${ }^{10}$ entre otros.

En tal situación sólo queda la opción de, o bien continuar peleando contra los molinos, aunque ya sepamos que no son gigantes, o bien hacer un planteamiento epistemológico constructivo, es decir, de posibilidad de producción de nuevo conocimiento que asume la mutua implicación entre sujeto y objeto. Como apunta Ibáñez, ${ }^{11}$ esta mutua implicación no sólo no es denostable sino que es la condición que ufunda la posibilidad del conocimiento y extiende el campo de la observación a la propia subjetividad».

Pero aun siendo una condición necesaria, no puede ser entendida como suficiente, pues, por sí sola, no garantiza la eficiencia de un conocimiento que podria ser perfectamente incomunicable. Hará falta que nos dotemos de ciertos controles procedimentales como garantía de comunicación y posibilidad de comprensión de lo conocido y como posibilidad, asimismo, de alcanzar el acuerdo intersubjetivo que permita avanzar colectivamente en el conocimiento.

10. Wagensberg, Jorge: 1985, «Ideas sobre ta complejidad del mundo" Superinfimos, 3, Barcelona, Tusquets.

11. Ibáñez, Jesús: 1979, Más allá de la sociologia, Madrid, Siglo XXI. 


\section{UN PRINCIPIO DE DEFINICION}

Una vez esbozados los puntos críticos de la práctica científica actual y apuntado el proceso por el que la metodología se ha visto aupada a un puesto central y decisivo, quizá conviene empezar a dilucidar cuáles son los rasgos constitutivos de esta metodología que aparece reclamada para funciones tan diversas.

Para realizar tal ejercicio vamos a centrannos en el terreno de las ciencias sociales, y más específicamente en la Sociología, aunque esto no significa que vayamos a cerrarnos la posibilidad de realizar los excursos necesarios a los demás campos científicos.

La primera constatación a hacet es la de que, efectivamente, resulta difícil, por no decir imposible, realizar una definición formal, sólida y consistente de lo que se entiende por metodología. Entre varias razones, debido a la diversidad de problemas que los sociólogos se plantean y a la diversidad de los niveles de reflexión en los que operan para afrontar tales probiemas. En definitiva, encontramos que tanto la vía de definición formal - lo que los metodólogos dicen- como la vía de definición sustantiva - lo que los sociólogos hacen $\cdots$ presentan grandes dificultades de sistematización.

De principio encontramos una utilización confusa de términos -método, tuétodos, metodología, técnicas- que no es sino el teflejo de la no tesolución, tanto a nivel individual como colectivo, del problema de la relación entre teoría e investigación. Nuestra perplejidad ante tal confusión corre pareja a la de Grawitz: ${ }^{12}$ "No puede uno sino maravillarse del extremo desorden en este campo. La mayoría de los autores distinguen el método de los métodos. Con todo, se encuentra este término utilizado para caracterizar unos procedimientos que se sitúan a niveles muy diferentes, en cuanto a su inspiración más o menos filosófica, a su grado de abstracción, su fnalidad más o menos explicativa, su acción en unas etapas más o menos concretas de la investigación y el momento en que se sitúan.» Resulta pues obligado considerar alguna vía de sistematización conceptual que permita un principio de ordenación.

Un primer principio ordenador es el nivel de reflexión en el que se desarrollan y producen los instrumentos analíticos. Bourdieu, Chamboredon y Passeton ${ }^{13}$ plantean la existencia de tres niveles de teflexión -epistemológico, metodológico y tecnológico- orientados a tres finalidades u obje-

12. Grawitz, Madeleine: 1975, Métodos y Técnicas de las ciencias sociales, 2 vols., Barcelona, Hispano-Europea.

13. Bourditeu, P., Chamboredon, J. C., Passeron, J. C.: 1976 (e.o. 1973), El oficio de sociólogo, Madrid, Sigio XXI. 
tivos diferentes: la conquista contra la ilusión del sabet inmediato, la construcción teórica del objeto y la comprobación empírica. Ibáñez ${ }^{14}$ plantea igualmente estos tres niveles, pero además los concreta en los ámbitos para los que resuttan pertinentes las preguntas: ¿Para qué se hace? ¿Por qué se hace así? ¿Cómo se hace? En ambos casos lo que resulta evidente de entrada es que ninguno de los niveles de reflexión 0 , mejor dicho, las prácticas correspondientes a ellos, pueden funcionar de modo independiente, aunque efectivamente tienen una autonomía relativa. No obstante son frecuentes las concepciones y prácticas en las que los métodos y las técnicas utilizadas son completamente ajenos a la actividad teórica y, en consecuencia, les es concedida una validez casi universal para no importa qué tipo de problema teótico se tenga planteado. La referencia tópica a la abusiva utilización de la técnica de encuesta nos evita mayor explicitación.

A partir de esta sistematización tenemos, pues, un principio de definición de la práctica metodológica como aquelia que tiene como finalidad la construcción teórica del objeto y que debe dar justificación de por qué se desarrollan los trabajos de investigación de una determinada manera entre las múltiples posibles.

Dicho de otta manera, la práctica metodológica debe tener como productos finales un objeto de investigación y un dispositivo analítico justifcado y estrechamente vinculado a aquél. A nuestro juicio, lo que puede resultar más clarificador de en qué puede consistir tal práctica metodológica es la caracterización, ni que sea mínima, de tales productos.

\section{Caracteristicas del objeto de investigación}

En primer Iugar hay que considerar la diferencia entre los objetos tal y como se expresan en la realidad y los objetos constitutivos del interés investigador del sociólogo. Con ello no hacemos sino apuntarnos a una ya bastante importante tradición en la Sociología que distingue entre cuestiones sociales y cuestiones sociológicas. El proceso de transformación de una categoría a la otra, que ha sido denominado de muy diversas maneras: reencantamiento, problematización, desenmascaramiento, complejización, etc., sería, pues, aquello que daría especificidad al trabajo de sociólogo y, sobre todo, diferenciaría la Sociología de la pura reelaboración del sentido común, sentando la posibilidad de producir un conocimiento «nuevo».

Una primera vocación de la práctica metodológica es, pues, la de la transformación de los objetos sociales en objetos sociológicos, es decir, el dimen-

14. Ibáñez, Jesús: 1979 (op. cit.). 
sionamiento de tales objetos en términos significativos que trasciendan la pura percepción «ingenua» socialmente determinada de los mismos.

Es esta misma característica de construidos y de no dados de los objetos de investigación la que nos da las pistas de su constitución: no externos al sujeto, teórico-abstractos y contextualizados. Vayamos por partes. Si el investigador es el que construye el objeto, parece obvio que su propia subjetividad está implicada en tal proceso. Muchos han sido los que desde la Sociología y otras ciencias, incluso naturales, han señalado la importancia de esta implicación, no ya como obstáculo tal y como se explica desde el paradigma dominante, sino como fundamento del conocimiento tal como hemos apuntado más arriba. A nosottos nos es especialmente grata la expresión del lingüista Saussure: ${ }^{15}$ «Es el punto de vista el que crea el objeto.»

Pues bien, una de las tareas primeras que debe afrontar la práctica metodológica es la de la explicitación y sistematización de la subjetividad del investigador, es decir, la base material sobre la que opera para construir su objeto. Sólo ello permitirá comprender el conocimiento producido en la medida que se sabrá no sólo lo que se dice sino, lo que es más importante, desde donde se dice.

La segunda dimensión constitutiva del objeto es la de su condición teórico-abstracta. Si el objeto de investigación ha de ser construido o producido por el investigador, quiere decir que su estructura sustantiva ha de ser una modelización, por lo tanto teórico-abstracta, dinámica del comportamiento simultáneo de los elementos intervinientes que el investigador determina como relevantes. Dicho de otra forma, la estructura del objeto no puede ser otra cosa que la propuesta teórica de un modelo de interacciones entre elementos significativos, o sea, un sistema de hipótesis. Desde esta perspectiva de la práctica metodológica el sistema de hipótesis no es algo adicional al objeto, sino que es básicamente la estructura del objeto de investigación.

$\mathrm{Y}$, finalmente, la tercera dimensión constitutiva del objeto es la de su naturaleza contextual. Decir que no puede darse la construcción de un objeto de investigación genérico, independiente del contexto social en el que se produce, es, de hecho, una trivialidad. Prácticamente la totalidad de los investigadores están de acuerdo en destacar la importancia del contexto en la definición del objeto. La pequeña diferencia está en que mientras para unos este contexto interviene como elemento modificador o mediador del objeto, sobre todo en la medida en que se trabaja con objetos sociales no sociológicos, para nosotros este contexto es constitutivo del objeto. El ob-

15. Saussure, Ferdinand: 1981, Curso de Lingüistica General, Madrid, Akal.

8. 
jeto de investigación descontextualizado no puede existir como objeto sociológico.

\section{Construcción del dispositivo analítico}

Según lo dicho anteriormente, el dispositivo analítico resultaría de una reflexión metodológica tendente a justificar por qué las cosas se hacen de un cierto modo. Peto resulta obvio que la justificación debe encontrar sus raíces en el propio objeto de investigación y no fuera de él.

En otras palabras, sólo en la medida que el investigador haya sido capaz de determinar la naturaleza del objeto o, lo que es lo mismo, el tipo de pregunta que pretende responder, estará en condiciones de articular un dispositivo de interrogación.

La catacterística básica de este dispositivo es la de responder sí o no, de confirmar o refutat el modelo teórico abstracto o el sistema de hipótesis, dígase como se quiera, construido con anterioridad. Bien es sabido que algunos investigadores cargan, a beneficio de inventario, sobre la cuenta del dispositivo analítico la posibilidad del «descubrimiento», cuando no han podido o sabido o, notmalmente, ni tan siquiera pretendido establecer las hipotesis de contraste. Esto equivale a pensar que la realidad por sí sola, interrogada por medio de mágicos procedimientos, escogidos de entre una panoplia cada vez más amplia y sofisticada, pero sin justificación teórica alguna que los refiera al objeto en cuestión, manifestará los rasgos constitutivos del problema y aun las posibles pautas de intervención.

El dispositivo analítico, fatalmente condenado a no ser justificado en términos de procedencia, sólo encuentra justificación en criterios mágicos, estéticos, de modernidad o de metcado y, en consecuencia, se ve abocado a una total esterilidad productiva de conocimiento nuevo.

La construcción de un dispositivo analítico es, pues, nada más y nada menos que la implementación de una lógica que justifica y escoge los posibles mecanismos de interrogación a la realidad para poder establecer la veracidad y eficiencia de nuestro modelo, de nuestro sistema de hipótesis. $\mathrm{Y}$ cuando decimos implementación lo decimos en sentido pleno. No nos olvidamos de las técnicas concretas de interrogación. Pero implementación de una lógica es más que la yuxtaposición de técnicas, aunque, por supuesto, éstas deben estar ahí.

Incluso sólo desde el punto de vista del dispositivo analítico, la metodología va más allá de la tecnología y de su know-bow. Otra cosa es que efectivamente la práctica metodológica exija el conocimiento de ambas para poder cumplir su función. 
En este sentido hay que temarcar que muchas veces se ha pervertido el debate metodológico reduciéndolo al debate sobre las técnicas o tecnologías que se utilizan. Hemos oído muchas veces los términos metodología cuantitativa y metodología cualitativa expresados como términos irreconciliables. Desde nuestro punto de vista esto es un falseamiento in nuce del problema y del debate. Expresado así es un falso problema. No existen metodologías cuantitativas o cualitativas. Existen metodologías con diverso grado de teflexión y de vocación de producción de nuevo conocimiento, con diverso nivel de problematización y que utilizan tecnologías diversas, a veces cuantitativas y a veces cualitativas.

No siendo éste el núcleo central de la cuestión, creemos que los principales problemas que hoy tiene la investigación sociológica por lo que se refiere a la construcción del dispositivo analítico son los referidos a la pro. ducción de la información y que, expositivamente, podemos dividir en dos cuestiones importantes: el problema de la observación y el problema de la medida. A ellos dedicamos el apartado siguiente.

\section{LA PRODUCCION DE DATOS EN LA INVESTIGACION}

A nuestro juicio ha de hacerse un esfuerzo importante por romper la cerrazón en que la metodología se ha visto inmersa por: el poso positivista de muchos investigadores, a saber, la preocupación exclusiva de extraer conclusiones legítimas a partir de los datos. Como muy bien dice Costner, ${ }^{16}$ «el problema de cómo producir los datos que se ban de utilizar continúa siendo un problema por tratar en la metodología sociológicas. A pesar de que ha transcurrido más de una década desde tal afirmación, pensamos que continúa siendo verdadera, y sólo si consigue resolver tal deficiencia podrá darse un avance cualitativo importante en el terreno de la metodología.

Resuita evidente que la solución a tal problema no es trivial y que hay que abandonar la perspectiva de una solución única en sentido operativo. Se podría afirmar que casi sería suficiente para dar este salto cualitativo el que los investigadores problematizasen la obtención de los datos necesarios por el simple procedimiento de definirlos y definir su grado de pertinencia a partir de la construcción sistemática de sus objetos de investigación.

Somos conscientes de que tal propuesta encuentra un obstáculo difícil de salvar en la aparente potencia de los métodos cuantitativos que boy se

16. Costnet, Herbert L. (ed.): 1971, Sociological Methodology 1971, San Francisco, Jossey-Bass. 
emplean masivamente en el trabajo de investigación. Parece imposible, y puede ser verdad hasta cierto punto por desconocimiento de los investigadores, discutir con tales métodos o alterar la lógica interna de tales artéfactos analíticos. Pero a nuestro juicio no es ésa exactamente la cuestión. Para nosotros, problematizar la obtención de datos quiere decir no renunciar a la lógica propia del análisis en favor de una lógica abstracta cristalizada en ciertas técnicas y, manteniendo la primacía de nuestra lógica, evaluar qué métodos de obtención de datos y de análisis de los mismos son los que mejor se adecúan a nuestro propósito.

Ello supone, entre otras cosas, entender que las técnicas de obtención y análisis de datos no son meros instrumentos empíricos, neutrales desde un punto de vista ideológico o de adscripción a ciertas teorías, sino que son «dispositivos prácticos de poder», es decir, que llevan incorporado un significado teórico preciso a partir del cual resultan pertinentes o impertinentes para una lógica de análisis concreta.

Como puede verse, hasta el momento no se han planteado consideraciones especiales para unos tipos de instrumentos analíticos $u$ ottos. Tanto aquellos con un nivel elevado de formalización como aquelios otros más informales deben estar sujetos a un mismo tipo de análisis crítico ante su eventual utilización. En nuestra opinión, aquellos más formalizados y que aparecen normalmente como neutros gracias a su extraordinaria divulgación son los que más lo requieren, puesto que, en general, la formalización sofisticada ayuda a enmascarar lo realmente sustantivo del instrumento.

\section{El problema de la abservación}

Respecto de las técnicas de obtención de datos hemos de decir que, en general, se ban construido bajo dos supuestos que resultan cuando menos discutíbles. El primero es el de la existencia de la información independiente del instrumento que la recoge y del sujeto que utiliza el instrumento; el segundo, que en cierta forma es su reverso, la preocupación por eliminar la contaminación o distorsión subjetiva de la información a recoger. Nuevamente encontramos un tremendo poso positivista en esta forma de entender la obtención de datos que, sin liegar a explicitarse como tal, constituye una forma de teorización «ingenua» del problema de la observación. Como ya apuntara en el siglo pasado Spencer, ${ }^{17}$ el problema de la observación tiene dos componentes básicas: $a$ ) el carácter poco perceptible de los fenómenos

17. Spencer, Herbett: 1969 (e.o. 1873), The Study of Sociology, Ann Arbor, The University of Michigan Press. 
sociales y b) los lazos emocionales indiscutibles que vinculan sujeto y objeto de conocimiento.

La pretensión de un registro neutro, sin supuestos, es algo hoy insostenible incluso en el terreno de las ciencias físico-naturales, y desde luego inaprovechable desde el punto de vista de la construcción de una teoría de la observación.

Por el contrario, ésta cada vez más necesaria teoría debe tomar en consideración que la observación es un proceso activo de producción de datos que supone en todos los casos, en mayor o menor medida, una intervención sobre lo observado que lo modifica.

A nuestro juicio el criterio teórico que debe presidir la construcción de una teoría de la observación es el expresado por Feyerabend, ${ }^{18}$ según el cual la realidad no es repetible ni teproducible, sólo reconstruible desde una posición teózico-analítica. Toda observación de la realidad supone un pro ceso de intercambio en el que, desde luego, resultan muy importantes tanto el posicionamiento teórico desde el que se dispone tal intercambio como el encuadramiento de lo intercambiado en el marco general de la investigación.

\section{El problema de la medida}

En esta línea, emerge con entidad propia el problema de la medida en Sociología, que, por otra parte, tampoco pensamos que sea muy diferente desde un punto de vista conceptual del problema de la medida en la ciencia en general. Ciertamente en el ámbito de las ciencias naturales rige desde hace siglo y medio aproximadamente un acuerdo — sistema métrico decimal- que obvia algunas cuestiones pero no otras. En particular no solventa la cuestión de una cierta perversión ligada a la medición. Kula ${ }^{19}$ cita las Antigüedades Judias de Flavio Josefo, en las que éste atribuye a Caín la invención de las medidas. Después de haber dado muerte a su hermano Abel, cometió ottos actos abominables, y entre ellos, «habiendo inventado pesas y medidas, transformó aquella inocente y noble naturalidad con que vivía la gente mientras las desconocía en una vida plena de estafas». Así pues, encontramos que la idea de la medición como generadora de «estafa» viene de antiguo.

Pero, más que recorrer la historia de la medida en Sociología buscando las «estafas» y perversiones con las que se corresponden, queremos apuntar

18. Feyerabend, Paul K.: 1977 (e.o. 1970), Contra el método, Barcelona, Ariel. 19. Kula, Witold: 1980 (e.o. 1970), Las medidas y los bombres, Madrid, Siglo XXI. 
dos ideas para nosotros fundamentales para una hipotética teoría de la medida que también está por construir.

En primer lugar, la constatación de que la medida, la escala con la que se pretende dimensionar ałgo externo al sujeto, jamás es aséptica y neutra. Siempte representa un valor. Por citat un ejemplo extremo, la operación de medida más simple, como es el recuento, utiliza una medida, los números naturales, que tiene de natural y neutra sólo su propia historia desde que fueron conceptualizados como tales y aun quizás menos, pues tardaton en ser impuestos y aceptados. Sabido es que los pitagóricos conceptualizaban números de muy diverso orden y que no utilizaban los dígitos arábigos que hoy se asocian convencionalmente a los números naturales. Sirva esta breve referencia para cuestionar la neutralidad de la medida y para asociar su nacimiento a los valores para los cuales resulta significativo aislar tal dimensión.

En segundo lugar, queremos destacar el hecho que, en la histotia de la humanidad, la fijación de medidas ha sido y continúa siendo un atributo del poder. Medir o ser medido representan posiciones diversas en las acciones de poder y todo es susceptible de medición excepto el propio poder, que siempre resulta inconmensurable. De alguna manera podemos decir que el sometimiento a medida de los objetos, y no digamos ya la construcción de tal medida, resulta un ejercicio de poder que puede resultar tanto más despótico cuanto menos respete las peculiaridades y el contexto del objeto.

Una teoría de la medida debe contemplar necesariamente el riesgo de despotismo en sus acciones, sobre todo porque tal despotismo condena al sujeto que lo ejerce a desconocer los objetos medidos.

$Y$ así, como el que no quiere la cosa, nos hemos introducido en el tema de la cuantificación en Sociología, que, sin lugar a dudas, es uno de los grandes temas y también una de las grandes servidumbres en la historia de la investigación sociológica.

La «magia» del número, la pretensión de dominio sobre los objetos de conocimiento a través de su representación numérica, es algo que bajo diferentes formas está presente en la Sociología desde sus orígenes. El tema es suficientemente conocido como pata poder ahorrarnos aquí una mayor explicitación de autores y obras.

Sin embargo, sí quisiéramos apuntar que hoy sigue siendo necesario cuestionatse el tema de la cuantificación, especialmente en un contexto en el que se ha producido una doble revolución: 1) Ia revolución de las computadoras, que permiten procesar y efectuar operaciones sobre cantidades tremendas de información en tiempos prácticamente despreciables, y 2) la apatición de las organizaciones de recogida sistemática de información, que 
son capaces de suministrarnos instantáneamente datos previamente tecogidos. Ambas suponen el alejamiento del sujeto de todo el proceso de producción de datos, tanto desde el punto de vista del desconocimiento de los procesos, cálculos y técnicas utilizados, muchas veces sin cuestionar su pertinencia y significación, como desce el de la utilización de una información recogida bajo una lógica diversa de la del propio análisis. Pero éste es un alejamiento taramente cuestionado y es mucho más frecuente entregarse a la seducción de lo que nos ponen fácil.

En nuestra opinión, una reflexión metodológica seria debe tender a reubicar la unidad central del proceso de producción de datos en el investigador y utilizar como periféricos, en algunos casos muy eficientes, los instrumentos tecnológicos derivados de esta doble tevolución.

Por otra parte, hay que reconocer que el desarrollo de periféricos inteligentes ha permitido avanzar mucho en la resolución de problemas tópicos que exan prácticamente irresolubles «a mano». En particular el tema de la semejanza y la clasificación, la construcción de grupos homogéneos, etc., ha podido ser asumído y resuelto de una forma más o menos eficiente a partir del desarrollo de los ordenadores. Hoy se está avanzando tremen. damente en los procesadores de textos y en los programas de teconocimiento de formas y perfiles, etc. Pero eso no significa que la lógica analítica pueda ser sustituida por la lógica de las máquinas, como sucede algunas veces por dimisión de los propios investigadores.

La informática y los sistemas de información pueden representar gran. des ayudas a la investigación a condición de que se haga de ellos un uso adecuado e inteligente, y eso exige evidentemente conocerlos. Despreciarlos o rechazarlos sin conocerlos es cuando menos tan tonto como aceptarlos sin cuestionamiento.

\section{LA DIMISION METODOLOGICA}

A lo largo de este texto ha salido algunas veces la palabra dimisión referica a aquellas actitudes y prácticas, conscientes o inconscientes, en las que el investigador hace renuncia de su propia centralidad en el proceso de investigación, sin que por ello renuncie formalmente a la importancia de la metodología. Actitudes y prácticas que pueden producirse aisłada o conjuntamente en diversos momentos de la investigación.

No tiene demasiado sentido, pues, reseguir todo el proceso arriba descrito para detallar todos y cada uno de los puntos en los que tal dimisión puede producirse, puesto que ello resultaría reiterativo. Por el contrario, 
puede resultar más ilustrativo, y seguro que de lectura más ligera, intentar tipificar algunos comportamientos, bien entendido que los tipos que a continuación se describen no suelen darse en estado puro entre los sociólogos, sino más bien como combinaciones y variaciones de ellos.

Las descripciones que siguen pretenden set un muestrario o bestiatio amable y no exhaustivo de especies sociológicas al uso catalogabies como «especies dimisionarias». Todas ellas se caracterizan por un rasgo dimisionario específico, si bien, como hemos dicho antes, lo normal es encontrar hibridaciones varias.

\section{Los ingenuos ingeniosos}

Su principal rasgo dimisionatio es el de no plantearse jamás que pueda haber una realidad más compleja que la que se manifiesta cotidianamente. Tratan principalmente de describirla de una manera ingeniosa de tal guisa que su discurso aparezca como original y novedoso, aunque de hecho no sea más que una sarta de trivialidades ya conocidas. Generalmente sus productos se caracterizan por no aportar nada nuevo al conocimiento e interpretación de los problemas, que, por lo común, son tomados directamente de la vida misma sin mediaciones intelectuales que los puedan enturbiar.

Son fervientes partidarios de la opinión de que la realidad habla por sí misma a condición de que sea interrogada sin trampa ni cartón, es decir, de modo neutral. Consecuentemente creen con firmeza en la separación radical entre sujeto y objeto de la investigación y, en su variante más ingeniosa, acostumbran a tener grandes éxitos de ventas.

Acostumbran a utilizar un lenguaje formalmente moderno y critico como apoyatura funcional para decir perogrulladas.

La subespecie de «no ingeniosos» son además insoportables.

\section{Los eruditos «a la violeta»}

Su principal característica es la de no definir jamás por sí mismos sus objetos de investigación. Se trata de una especie más taimada que la anterior, puesto que toman en préstamo, como sustitutivo del proceso de construcción, definiciones elaboradas por otros, para otros objetivos y otras finalidades, que casi nunca especifican.

Su gran recurso es la cita erudita (con una media estimada entre 3 y 5 citas por página), que utilizan como elemento fundamentalmente defen- 
sivo frente a eventuales ctíticas. Acostumbran a tener un gran dominio de la técnica de la analogía, con la que obran auténticas maravillas para ofrecer lecturas novedosas. Su gran problema es que finalmente suele quedar bastante claro que no saben de qué están hablando, pues les acostumbran. a fallar los cimientos conceptuales sobre los que construir, además de manejarse habitualmente con materiales de derribo, de desecho o impropios, que de todo hay en la viña del Señor.

En general, suelen ser bastante superficiales - de ahif lo de «a la violeta»- $\rightarrow$ aunque últimamente se está prodigando bastante la subespecie embolica que fa fort, que consigue ocultar bastante bien la superficialidad bajo una maraña espectacular, especular y heterogénea de citas y referencias.

Suelen vivir a la sombra institucional y no tienen grandes éxitos de. ventas, aunque, por lo general, tienen bastante predicamento académico.

\section{Los fascinados}

Su característica más definidora es la de haberse quedado prendidos. en una idea - a veces una tecnología específica, que de todo hay- y se limitan a reducir todo su ámbito de preocupación a la reproducción, algunas veces ampliada, de dicha idea o tecnología.

Acostumbran a ser fanáticos y dogmáticos, aunque no ignorantes, y ven a los discrepantes como auténticos enemigos a los que hay que combatir en todos los terrenos. Quizás porque la mera existencia de «diferentes» pone en peligro su propia seguridad. Son básicamente inseguros.

Suelen ser teduccionistas y cualquier elemento de la tealidad les sirve como pretexto para acabar hablando de lo que siempre hablan o haciendo lo que siempre hacen.

Suelen ser de corta vida pública si no se reconvierten, aunque hay casos de longevidad y éxito profesional -normaimente allende las fronterasadmirables.

Se da una subespecie bastante dañina constituida por individuos fascinados por la idea de la negación y/o de la cotrupción. Pata ellos todo lo que bacen los demás es negativo y cotrupto y suelen acabar generando paranoias, lo que los hace francamente inaguantables para vivir en comunidad con otras especies. No obstante se toleran bastante bien con los maniqueos debido a sus comunes aficiones depredadoras. 


\section{Los maniqueos}

Como apuntábamos antes, tienen rasgos comunes con los fascinados negativos, aunque los diferencia el hecho de estar mucho más seguros de sí mismos. La depredación suele ser en estos individuos un brillante ejercicio de demostración de lo mucho que valen. Su característica dimisionatia es la de no cuestionar jamás su propia posición frente a los objetos, muchas veces completamente visceral, lo que se complementa con la trivialización $\mathrm{y}$ desprecio de los argumentos o posiciones discrepantes.

Suelen ser hábiles argumentadores $\mathrm{y}$, como rasgo específico diferenciador de los anteriores, tienden a amalgamat todo lo foráneo en una misma cosa, a la que tienden a calificar de perversa.

En general son poco agresivos, aunque si se les provoca pueden resultar altamente peligrosos, pues es sabido que no hay nada tan peligroso como un individuo poseedor de la verdad o, lo que es peor, convencido de ello.

Suelen ser de producción corta y monotemática y muestran una tendencia exagerada a vivir en pequeñas colonias de adictos.

\section{Los bijos del situacionismo}

Aunque el situacionismo tuvo clatamente definida desde el principio su condicion de efimero, ha generado post mortem una especie que se caracteriza por intentar perpetuar, con carácter general, los rasgos de provisionalidad e interinidad, lo que no deja de ser una paradoja interesante. Así, el análisis concreto de situaciones concretas se ha convertido para los individuos de esta especie en un nuevo paradigma limitado estrictamente a la interpretación de fenómenos particulares.

Aunque no lo manifiestan, su rasgo dimisionario más claro pasa por la renuncia a la posibilidad de comunicación de lo conocido, la tenuncia a lograr un acuerdo intersubjetivo y, consecuentemente, renuncian a la eficiencia instrumental del conocimiento.

Suelen invocar la teoría psicoanalítica y a los etnometodólogos y muestran una afición especial por los problemas individuales o de pequeños grupos, que, según algunos, aunque no está confirmado, no revela otra cosa más que la transferencia hacia lo público de sus propias problemáticas individuales o grupales.

Su instrumento preferido es la especulación, y entre ellos hay auténticos maestros especuladores. Tienen concomitancias con algunas especies de Ias faunas antropológica y filosófica, aunque por otra parte se suelen mostrar celosos guardianes del corporativismo sociológico.

Muestran un cierto carácter gregario y no son agresivos. 
Los «No me lo cuentes, vecina»

De las especies dimisionarias, ésta es la que está constituida por los individuos más simpáticos y felices. Acostumbran a manifestar ostensiblemente su perplejidad ante cualquier complejidad que se les plantee y prefieren no enterarse de la misma, reenviándola a los campos competenciales correspondientes, que, por supuesto, nunca son los suyos.

Su configuración dimisionaria es total y su instrumento preferido es el manual. Suelen fabricat productos reader's digest y acostumbran a tener cierto éxito profesional entre los consumidores de sociología wash \& wear.

Formalmente se reclaman interdisciplinarios, posiblemente por la necesidad que experimentan constantemente de relacionarse con expertos de los diferentes campos y temáticas complejas con los que se enfrentan.

Acostumbran a ser público agradecido y gran consumidor de charlas, seminarios y conferencias, no porque tengan gran interés en complicar su cuadto teórico referencial, sino porque esa actividad les permite detectat presuntos especialistas y ampliar su ted de conexiones interdisciplinares.

En cualquier caso, son extraordinariamente sociables.

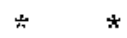

Damos por finalizado, por el momento, el bestiario amable de especies sociológicas dimisionarias. Esperamos que se entienda que las diferentes especies mostradas no pretenden cotresponderse con ningún personaje en concreto, aunque no nos es lícito decir aquello tan sobado, pero mono al frn y al cabo, de que cualquier parecido con la realidad es pura coincidencia.

De hecho, lo que hemos pretendido es ofrecer una serie de reflexiones sobre nuestra propia práctica como investigador y conjurar a nuestros propios fantasmas para que cada cual pueda explorat sus propios límites. Sólo así seremos todos capaces de dar un saito adelante, que buena falta nos hace. 